\title{
Long-term hydrophilization of polydimethylsiloxane (PDMS) for capillary filling microfluidic chips
}

\author{
Farzin Jahangiri $^{1}\left[\right.$ ] Tuuli Hakala $^{1} \cdot$ Ville Jokinen $^{1}$
}

Received: 16 April 2019 / Accepted: 5 November 2019 / Published online: 22 November 2019

(c) The Author(s) 2019

\begin{abstract}
We present a simple and facile method for long-term preservation of hydrophilicity of oxygen plasma-hydrophilized poly (dimethylsiloxane) (PDMS) by cold storage. We show that storage under temperature of $-80^{\circ} \mathrm{C}$ can maintain superhydrophilicity of plasma-exposed PDMS for at least 100 days. Storage at $-15^{\circ} \mathrm{C}$ and at $22{ }^{\circ} \mathrm{C}$ room temperature (RT) is shown to exhibit, respectively, about half and full recovery of the original hydrophobicity after 100 days in storage. Furthermore, we investigated the implications of the cold storage for microfluidic applications, the capillary filling rate and the ability of the flow to bypass geometrical obstacles in a microfluidic channel. It is shown that the preservation of capillary filling properties of microchannels is in close agreement with the contact angle (CA) measurements and that the colder the storage temperature, the better the capillary filling capability of the channels is preserved. We ascribe the significantly reduced recovery rate to reduced thermally activated relaxation phenomena such as diminished diffusion of low molecular weight species (LMW) in the polymer matrix at colder temperatures. This is supported by ATR-FTIR measurements of the $\mathrm{OH}$ vibration band over time for samples stored at different temperatures.
\end{abstract}

Keywords PDMS $\cdot$ Hydrophobic recovery $\cdot$ Cold storage $\cdot$ Long-term hydrophilicity $\cdot$ Capillary microfluidics $\cdot$ Geometrical valves $\cdot$ ATR-FTIR analysis

\section{Introduction}

Over the last two decades, microfluidic systems have witnessed a rise in their applications in life sciences, chemistry and diagnostics (Duncombe et al. 2015; Elvira et al. 2013; Sackmann et al. 2014). Microfluidics offers enhanced fluidic control and precision over metering and monitoring. Soft lithographic methods (Xia et al. 1998) have made microfluidic devices more and more accessible to the end users.

Capillary action is the spontaneous movement of fluid on the surface of a solid material due to surface energy minimization. Capillary-driven systems allow even complex fluidics operations to be programmed on the chip by designing the geometry and the surface energy of the chip (Juncker et al.

Farzin Jahangiri

farzin.jahangiri@aalto.fi

Ville Jokinen

ville.p.jokinen@aalto.fi

1 Department of Chemistry and Materials Science, School of Chemical Engineering, Aalto University, Aalto, 00076 Espoo, Finland
2002; Safavieh and Juncker 2013; Mosadegh et al. 2010). Among physical parameters used to characterize capillary filling of microchannels, contact angle (CA) on the open surface in a three-phase system and capillary filling dynamics in closed micro-geometries are the most widely used (de Gennes 2004).

Microfluidic chips are nowadays most commonly made from polymer materials due to their ease of processability, low cost of fabrication and ability to conformally replicate any premade micro- and nano-structures. The most widely used polymer for microfluidics, poly (dimethylsiloxane) (PDMS) is inherently hydrophobic. In fact, most of the available polymers have either hydrophobic or only slightly hydrophilic surface chemistries, which provide insufficient capillary pressure for capillary driven systems.

Because of this, a multitude of methods have been employed to render hydrophilic properties onto these surfaces to enable bio- and capillary filling-based applications (Ferreira et al. 2015; Yoshida et al. 2013; Tsougeni et al. 2009; Khorasani et al. 2008; Vlachopoulou et al. 2009; Tan et al. 2010). Plasma treatments are a widely used technique to increase the surface energy of polymers (Tan et al. 2010; 
Jokinen and Franssila 2008). However, a near universal observation is a hydrophobic recovery that partially or completely negates the effect of the plasma hydrophilization over time (Owen and Smith 1994; Jokinen et al. 2012).

In general, three mechanisms have been proposed to contribute to hydrophobic recovery of PDMS: Bulk to surface migration of the low molecular weight species (LMW) (Toth et al. 1994; Hillborg et al. 2000, 2004), surface re-structuring phenomena including re-orientation of hydroxyl groups toward bulk due to energetically unfavorable arrangement and condensation of silanol groups on the surface (Morra et al. 1990; Bacharouche et al. 2013), and generation of LMW species within the surface during the plasma treatment (Toth et al. 1994; Kim et al. 1999, 2001a, b). Process parameters which significantly influence the contribution of such mechanisms include pre-polymer composition, curing temperature and time, sample thickness, vacuum treatment, plasma treatment parameters, post-plasma modifications and sample storage conditions (Nguyen 2014). Numerous efforts have been conducted on reducing, if not completely eliminating, the recovery over time by focusing on one of those process parameters.

Eddington et al. (2006) reduced the recovery rate by reducing the primary cause of it, i.e., removing free oligomers, through polymerizing uncrosslinked monomers by means of extended thermal aging of the PDMS. Solvent extraction by heptane prior to plasma introduction rendered a $5 \mathrm{wt} \%$ weight loss of PDMS samples (Lee et al. 2003) and led to reduced hydrophobic recovery for a week when stored in air at room temperature (Vickers et al. 2006). Nguyan et al. (2014) examined the effect of the prepolymer composition on durability of hydrophilization. By removing cyclic dimethylsiloxane groups (predominant LMW moieties responsible for recovery) from the mix through extended room temperature curing and longer vacuum steps of $40 \mathrm{~min}$ prior to plasma treatment, they achieved low dynamic contact angles of $\theta_{\mathrm{A}} / \theta_{\mathrm{R}}=51^{\circ}-56^{\circ} / 38^{\circ}-43^{\circ}$ on silicones measured more than 30 days after the plasma treatments. Hemmilä et al. (2012) studied the effect of various surface treatments on PDMS by plasma-activated physisorption, grafting to-and-from with biocompatible HEMA, PEG and PVA polymer brushes and could retain hydrophilicity of some of the samples up to 6 months. By introducing a plasma-activated two-step grafting of PEG polymer brushes, Delamarche et al. (2003) achieved hydrophilic stability of PDMS stamps suitable for micro-contacting printing $(\mu \mathrm{CP})$ of polar inks. Layer-by-layer (LbL) deposition of counterions in a sequential manner on freshly oxidized PDMS has also been employed for stable hydrophilization of the microfluidics toward droplet generation (hexadecane in water) which maintained droplet generation ability up to 5 months (though no capillary data available) (Bauer et al. 2010). Storage of samples in polar solvents (deionized water and
Luria-Bertani broth) has been shown to postpone recovery up to a week as hydrophilic surface groups energetically favor retaining the surface position (Zhao et al. 2012). Bulk modification of the PDMS with amphiphilic surfactant PEO poly(dimethylsiloxane-ethylene oxide polymeric) has been shown to impart a tunable hydrophilicity $\left(21.5^{\circ}-80.9^{\circ}\right)$ ratio up to 2 months with a consequent fade away (Yao and Fang 2012). A two-step process including UV treatment of PDMS combined with PVA surface coating after plasma treatment (Bodas and Khan-Malek 2006) allowed recovery of contact angle up to $70^{\circ}$ in 2 weeks. Solvent extraction by trimethylamine, ethyl acetate and acetone in sequence (Vickers et al. 2006) maintained contact angles of $30^{\circ}-40^{\circ}$ up to a week. Preservation of hydrophilic surfaces in the presence of polar liquids (Ren et al. 2001) led to maintenance of electroosmotic flow after 14 days by immersion in water. Surface nanotexturing of PDMS by a two-step process of SF6 and $\mathrm{O}_{2}$ plasma in a sequential manner has also been explored and holds potential for maintaining hydrophilicity for up to 7 days (Vlachopoulou et al. 2009).

In the works discussed above, the sought after prolonged hydrophilization requires a physical or chemical intervention with footprints that might not be desirable for intended applications, commonly requiring a post processing step after hydrophilization. In this report, we show the hydrophilic state of PDMS can be maintained long term without a need of any invasive chemical and physical steps after the hydrophilization. Diffusion to and from surface, and reorientation of surface polar groups toward bulk are known as key mechanisms responsible for surface restructuring and hydrophobic recovery (Kim et al. 2001a, b). Leveraging the temperature dependency of the main recovery mechanisms, we demonstrate the superhydrophilicity of PDMS remains at least after 100 days by storing the samples in a sufficiently cold temperature. Freshly plasma-hydrophilized chips are stored in $-80{ }^{\circ} \mathrm{C},-15^{\circ} \mathrm{C}$ and room temperature (RT), and it is demonstrated that increasingly colder storage temperatures lead to significant retainment of the contact angle, capillary filling rate, and the ability of capillary flow to bypass geometrical obstacles in microchannels. These characteristic parameters are shown to correlate with surface chemistry, i.e., presence of $\mathrm{O}_{2}$ plasma-induced hydroxyl groups recorded by ATR-FTIR time-series measurement of the samples during the recovery.

\section{Materials and methods}

\subsection{PDMS fabrication}

PDMS sheets used for the sessile droplet goniometry measurements were prepared from Sylgard 184, Dow Corning by mixing a 10:1 ratio of monomer to crosslinking agent, 
degassing and planarizing prepolymer to $3-\mathrm{mm}$ thickness in a polystyrene petri dish. The samples were cured in an oven at $70{ }^{\circ} \mathrm{C}$ for $2 \mathrm{~h}$ and cut to test pieces of $2 \mathrm{~cm}^{2}$. Cleaning step was performed by immersing the PDMS pieces in isopropylalcohol (IPA) and sonicating for $45 \mathrm{~min}$, rinsed with DI water, dried by nitrogen gun and degassed in a fume hood overnight to remove absorbed IPA. Finally, the pieces were thermally aged in an oven at $100{ }^{\circ} \mathrm{C}$ for $51 \mathrm{~h}$.

The capillary filling experiments were made with PDMS microfluidic chips. The PDMS chips were made by replication molding from SU-8 masters. The SU-8 masters were made by spin coating SU-8 50 (Microchem) $2050 \mathrm{rpm} 45 \mathrm{~s}$, soft bake on a hotplate at $95{ }^{\circ} \mathrm{C}$ for $5 \mathrm{~min}$, UV exposure by mask aligner (model MA-6, SÜSS MICROTEC AG) for $10 \mathrm{~s}$, and post exposure bake at $95^{\circ} \mathrm{C}$ for $10 \mathrm{~min}$. PDMS was cast and cured with the same parameters as for the PDMS sheets. Flat sheets of 3-mm-thick PDMS as substrates for the channels, were likewise prepared in flat petri dishes. After curing, the chips were cut by a surgical grade blade and inlet and outlets were punched into the chips. Cleaning and thermal aging of the chips and substrates were carried identically to the PDMS sheets. The chips and the sheets were both exposed to oxygen plasma and afterwards placed in contact with each other to bond them covalently.

\subsection{Plasma treatment}

Plasma treatment of the PDMS sample sheets and microfluidic capillary chips was performed by Plasma System 400 batch reactor (PVA Tepla AG). Microwave power of $50 \mathrm{~W}$ $(2.45 \mathrm{GHz})$ was used to generate the plasma. Oxygen flow was set to $500 \mathrm{ml} / \mathrm{min}$ and the treatment time was $2 \mathrm{~min}$.

\subsection{Cold storage}

Individual PDMS sheets and microfluidic chips were stored in separate small petri dishes, divided into three equal batches, each of which to be maintained at a different storage temperatures (RT, $-15{ }^{\circ} \mathrm{C}$ and $-80^{\circ} \mathrm{C}$ ). For each data point reported in CAs at a given temperature, two sheets with three measurements on each were used and average $\mathrm{CA}$ and combined variance were reported as the standard deviation. In the capillary filling kinetics measurements, three batches of microchannel chips were prepared, each stored at a different temperature. For each of the reported data points representing a Washburn's constant (and corresponding calculated dynamic CA), a video recording of filling one complete channel at a specific day was acquired. For infrared vibrational spectroscopy experiments, for each data point on the results, three samples were measured and averaged over values. Geometrical valve measurements include three identical batches stored at three different temperatures. Each experiment involves the video recording of filling of one channel with five consecutive valves (increasing angle of widening) and results are reported as binary passage or not passage through valves at different days. A household refrigerator-freezer (Bauknecht) was used for $-15^{\circ} \mathrm{C}$ storage and a laboratory freezer (Thermo, Electron Corporation) was used for storage at $-80^{\circ} \mathrm{C}$. The samples were individually stored at the designated temperature in closed petri dishes until they were used in measurements. All samples did not spend time in room temperature air for longer than $30 \mathrm{~min}$, to minimize the thermal effects. Samples were thermalized to RT before measurements by ambient exposure.

\subsection{Contact angle measurement}

Advancing and receding contact angles were measured by the sessile droplet method (Theta, Attension). The droplet rate was $0.1 \mu \mathrm{l} / \mathrm{s}$ and the advancing and receding angles were measured between droplet sizes $2 \mu \mathrm{l}$ and $10 \mu \mathrm{l}$. The data points reported are the averages of six measurements ( 2 different sheets prepared with the same parameters and three points per sheet) with standard deviations calculated from combined variances. In cases where the determination of advancing or receding contact angles was not possible due to them being too low to be measured by sessile droplet goniometry, they were counted as $5^{\circ}$ in the results.

\subsection{Capillary filling rate experiment}

A meandering microchannel with extended straight sections (width $100 \mu \mathrm{m}$, height $51 \mu \mathrm{m}$, total length $10 \mathrm{~cm}$ ) was utilized in the capillary filling rate experiments. A batch of PDMS microchannel chips were stored in each of the storage temperatures. For each of the data points on the results, a single chip was taken out of the storage for measurements at designated days during a 2-week span. Capillary filling experiments were performed by pipetting $10 \mu \mathrm{l}$ of a mixture of water and food dye into the inlet, and recording a video of the filling process. The capillary filling rate was determined from these videos. During each filling experiments, a new timepoint was registered every time meniscus proceeded additional $10 \mathrm{~mm}$. The distance was measured by microfabricated markers on the chip (every $10 \mathrm{~mm}$ ). The experiment was a time series with a single data point for each storage temperature and each timepoint.

\subsection{Geometrical valve experiment}

A straight microchannel (width $100 \mu \mathrm{m}$, height $51 \mu \mathrm{m}$, length $2 \mathrm{~cm}$ ) with five sequential and axisymmetric geometrical widenings in the horizontal plane was used for testing the ability of capillary flow to bypass local geometrical barriers. The widening angles on each side of channel from smallest to biggest include $10^{\circ}, 40^{\circ}, 60^{\circ}, 80^{\circ}$ and $90^{\circ}$. 
Geometrical barrier passage experiments were performed by pipetting $10 \mu \mathrm{l}$ of a mixture of water and food dye into the inlet and recording a video of the filling process. The passage of the liquid through the barriers was determined from these videos as a binary decision. The results are based on a single sample per storage temperature and timepoint.

\subsection{ATR-FTIR}

An Attenuated Total Reflection Fourier Transformed Infrared apparatus, Spectrum Two ${ }^{\circledR}$ Perking Elmer with $\mathrm{KBr}$ windows for data collection was used for surface analysis. Spectral range of $4000-350 \mathrm{~cm}^{-1}$ at resolution of $4 \mathrm{~cm}^{-1}$ and four scans were used. A broad peak at around $3000-3500 \mathrm{~cm}^{-1}$ as a telltale of the $\mathrm{OH}$ bond vibrations (Bodas and Khan-Malek 2006) was selected for characterizing the hydrophilization and hydrophobic recovery. After the plasma treatment, the samples were tracked over 2 months. All OH peak heights were measured against their respective flat region in pristine PDMS (3000-3500 $\mathrm{cm}^{-1}$ ) and normalized to the first (highest peak) data point to highlight the relative strength of the signal on a particular point in time. Three identical samples were used for each peak measurement at a specific timepoint and temperature, and the average value of peak intensity was utilized for analysis to increase the statistical significance of the result. The plasma treatment duration applied to samples analyzed by ATR-FTIR was longer and about $30 \mathrm{~min}$, compared to the plasma time used for the contact angles and capillary filling measurements. The reason for this choice was that the 2-min treatment time did not produce a strong enough signal to be reliably detected by ART-FTIR.

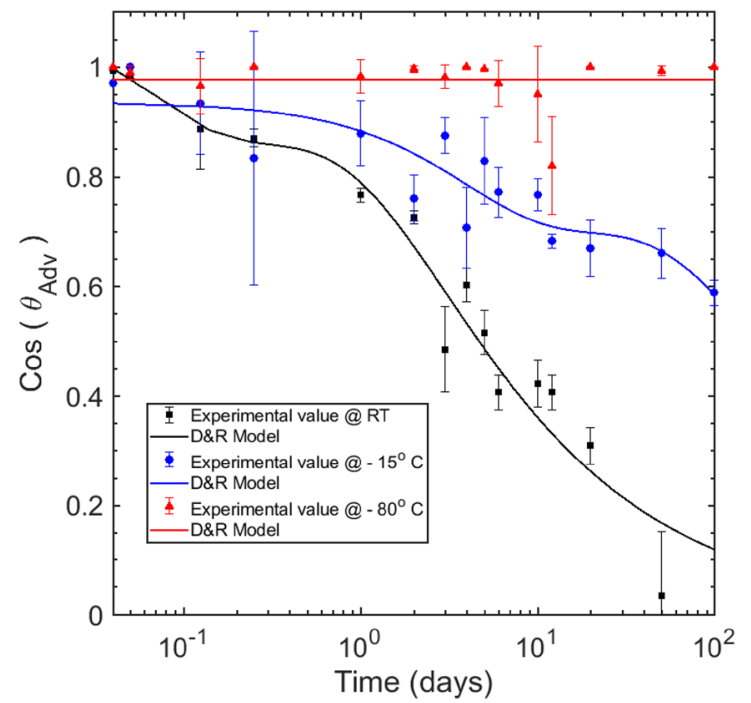

Fig. 1 Hydrophobic recovery of plasma-treated PDMS sheets as a function of storage temperature for advancing contact angle (left plot) and receding contact angle (right). Each data point is the average \pm of the combined variance of 3-6 measurements performed on two

\section{Results and discussion}

\subsection{Hydrophilization and hydrophobic recovery- PDMS sheets}

The resulting rate of hydrophobic recovery of plasmaexposed PDMS sheets stored at three different temperatures from the onset of hydrophilization up to 100 days for both the advancing and receding contact angles is shown, respectively in Fig. 1a, b. The contact angle of the samples stored in different temperatures separates clearly into three distinct trajectories over the span of 100 days. The $-80^{\circ} \mathrm{C}$ samples kept a steady cosine values of close to 1 , samples kept in $-15{ }^{\circ} \mathrm{C}$ followed a gradual drop to 0.6 and samples kept at RT reached a near 0 value for advancing CAs by the end of the 100 days. The native PDMS showed an advancing cosine value of $-0.52\left(\theta_{\mathrm{adv}}\right.$ of $\left.122^{\circ} \pm 7^{\circ}\right)$ and for receding cosine 0.01 (contact angle of $89^{\circ} \pm 2^{\circ}$ ). Immediately after the treatment, the PDMS became superhydrophilic corresponding to 0.99 in cosine value (advancing contact angle $7^{\circ} \pm 6^{\circ}$ and receding contact angle $\left.5^{\circ} \pm 2^{\circ}\right)$. The sample stored in the room temperature recovered to approximately cosine value of $0.77 / 0.84$ (contact angle $\left.\theta_{\text {avd }} 40^{\circ} / \theta_{\operatorname{Rec}} 33^{\circ}\right)$ in a day, $0.36 / 0.5\left(\theta_{\text {adv }} 69^{\circ} / \theta_{\operatorname{Rec}} 60^{\circ}\right)$ in a week and had a near full recovery to -0.14 and $0.28\left(\theta_{\mathrm{adv}}\right.$ $98^{\circ} / \theta_{\operatorname{Rec}} 74^{\circ}$ ) over 100 days. The sample stored at $-15^{\circ} \mathrm{C}$ recovered to approximately $0.85 / 0.93\left(\theta_{\text {adv }} 32^{\circ} / \theta_{\operatorname{Rec}} 21^{\circ}\right)$ in a day, $0.72 / 0.83\left(\theta_{\mathrm{adv}} 44^{\circ} / \theta_{\operatorname{Rec}} 34^{\circ}\right)$ in a week and settled to $0.56 / 0.46\left(\theta_{\mathrm{adv}} 56^{\circ} / \theta_{\mathrm{Rec}} 46^{\circ}\right)$ over 100 days. The sample stored at $-80{ }^{\circ} \mathrm{C}$ remained very hydrophilic throughout

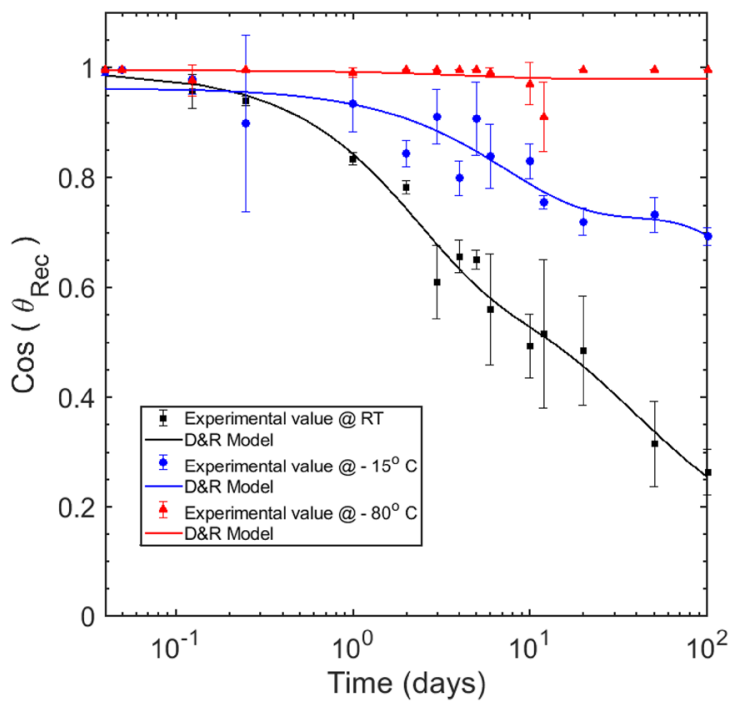

samples. The trend lines represent a kinetic recovery of diffusion and reorientation model, fitted against data, and further elaborated in the Eq. 1 
the experiment. For contact angles of most test pieces stored at $-80{ }^{\circ} \mathrm{C}$ the cosine values were more than 0.97 (less than $\mathrm{CA} 15^{\circ}$ ) throughout the experiment. Few of the data points have a large standard deviation. Experimental artefacts such as local heterogeneities of plasma treatment and surface contamination can be the origin of such large variations. However, the overall trends in both the advancing and receding contact angles are very clear.

A time-dependent model for hydrophobic recovery of polymers accounting for both LMW species diffusion and surface bound rotations, Diffusion and Rotation (D\&R) (Mortazavi and Nosonovsky 2012) is used to fit the data by a nonlinear least square solver in Matlab and is overlayed in Fig. 1. The full model accounts for surface concentration of hydrophilic moieties and their change over time, and relates the concentrations to contact angle through Cassie composite wetting model. The model is described by Eq. 1

$\cos (\theta)=C_{0}\left(c_{1} \operatorname{erf}\left(\frac{\delta}{2 \sqrt{D t}}\right)+C_{2} e^{-\frac{t}{\tau}}\right)\left(1-\cos \theta_{\mathrm{u}}\right)+\cos \theta_{\mathrm{u}}$,

where $C_{0}$ is the initial surface coverage of the polar groups after plasma treatment (equal to 1 for superhydrophilic surface), $\delta$ is depth of plasma treatment into the polymer, $D$ is the diffusion coefficient of LMWs, $t$ is the time, $\tau$ is the relaxation time of reorientation and $\theta_{\mu 1}$ is the native contact angle of polymer. The term $\left(\frac{\delta}{2 \sqrt{\pi D}}\right)^{2}$ is the characteristic time of the diffusion process and is extracted from the model as a single parameter.

The characteristic time scales for both diffusion and re-orientations can be extracted from the model when the input is a time series of contact angles. The diffusion and re-orientation characteristic times contribute to the overall rate of recovery. The diffusion characteristic times computed from our CA data at different storage temperatures suggest that recovery to the same degree requires 1.55 days, 97 days and 3400 days, respectively, for samples kept at RT, $-15^{\circ} \mathrm{C}$ and $-80^{\circ} \mathrm{C}$. The reorientation relaxation times are shortlived compared to diffusion times and not consequential for long-term hydrophilicity. For the first two computed diffusion characteristic times, respectively, occurring at room temperature and $-15{ }^{\circ} \mathrm{C}$ storage, data in our measurement range show a good agreement against the obtained parameters. The diffusion relaxation time for samples at $-80{ }^{\circ} \mathrm{C}$ storage cannot be empirically verified, as our experiments only lasted 100 days. However, qualitatively, it matches the expected large delays in recovery at $-80^{\circ} \mathrm{C}$.

Overall, the result is clear that the colder the storage temperature, the slower the hydrophobic recovery. This general temperature-dependent behavior is in accordance with the previously reported Arrhenius interpretations of PDMS hydrophobic recovery (Bauer et al. 2010).

\subsection{Attenuated total reflection Fourier transformed infrared spectroscopy}

Time-series results of ATR-FTIR measurements on flat PDMS samples showing the chemical composition of the PDMS surface during 10 weeks after the plasma treatment are presented in Fig. 2a. The evolution of the $\mathrm{OH}$ vibration peak (3000-3700 $\mathrm{cm}^{-1}$ ) intensities for each of the three temperatures is shown in the Fig. $2 b$, showing less change over time for the colder temperatures. Figure $2 \mathrm{c}$ shows the intensity of the $\mathrm{OH}$ peak as a function of recovery time and temperature. It is clear that three distinct trajectories emerge corresponding to the storage temperatures. The $\mathrm{OH}$ signal for room-temperature-stored samples drops to $60 \%$ in $24 \mathrm{~h}$ and continues a gradual decrease, with a $30 \%$ intensity in the end of measurement period. For samples stored at $-15{ }^{\circ} \mathrm{C}$, a drop to around $70 \%$ is recorded within $24 \mathrm{~h}$, following a steady decrease to final relative intensity of $37 \%$ by the day 72 . The intensity for samples kept at $-80{ }^{\circ} \mathrm{C}$ drop to $80 \%$ in $24 \mathrm{~h}$ and stay relatively constant afterwards with the final value of $55 \%$.

\subsection{Capillary filling rate}

The capillary filling rate of laminar flow in channels with constant cross sections obeys the Washburn relation, which can be written in the form given by Eq. 2 (Washburn 1921)

$L^{2}=R t=\frac{C \gamma \cos (\theta)}{\mu} t$,

where $L$ is the distance filled (location of the filling front), $t$ is the time, $\gamma$ is the surface tension, $\theta$ is the advancing contact angle, $\mu$ is the dynamic viscosity and $C$ is a factor that is dependent on the cross-sectional geometry. The parameter $R$ has units of $\mathrm{mm}^{2} \mathrm{~s}^{-1}$ and can be considered to be the capillary filling rate. First, we calculated the Reynolds number $\operatorname{Re}=\rho v l \mu^{-1}$, where $\rho$ is the density, $l$ is the characteristic dimension of channel and $v$ is the meniscus velocity during the fastest possible flow (freshly hydrophilized samples, first $10 \mathrm{~mm}$ of the filling). The maximum Reynolds number in the experiments was 0.455 which means that the flow is laminar and well within the Washburn regime.

Figure 3 a presents evolution of $R$ and corresponding calculated $\cos (\theta)$ of a microchannel (width $100 \mu \mathrm{m}$, height $51 \mu \mathrm{m}$ ) over time as function of the storage temperature. Figure $3 \mathrm{~b}$ depicts the schematics of the capillary filling channel. Freshly hydrophilized channels had a capillary filling rate of $83 \mathrm{~mm}^{2} \mathrm{~s}^{-1}$ (maximum linear velocity of $68 \mathrm{mms}^{-1}$ ). It is noticeable that the trends of filling rate for samples kept at three different temperatures exhibit 
(a)

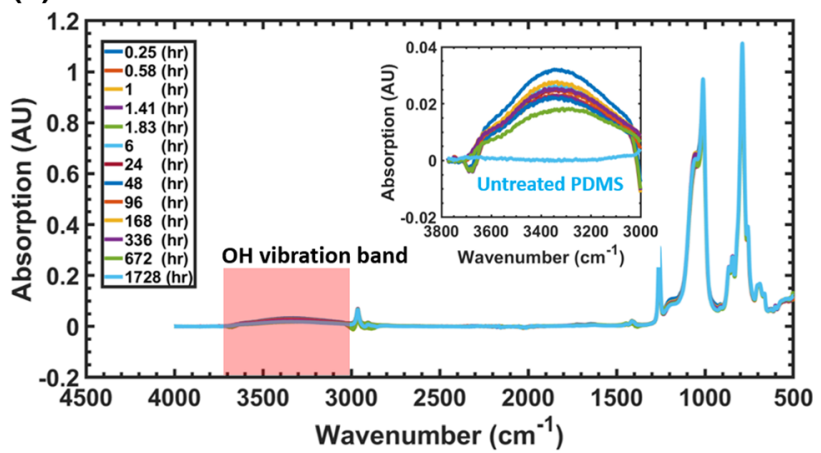

(b)

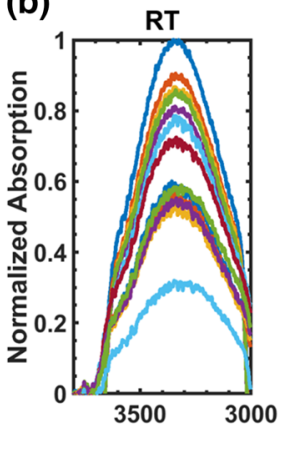

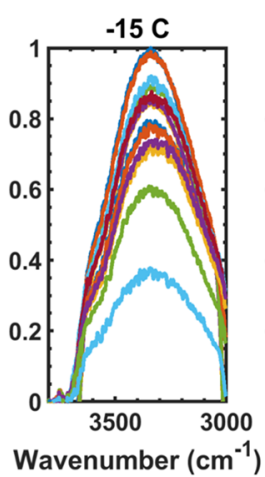

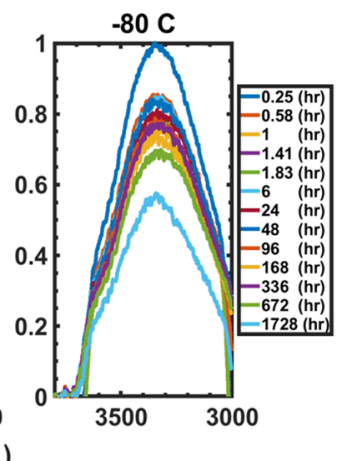

(c)

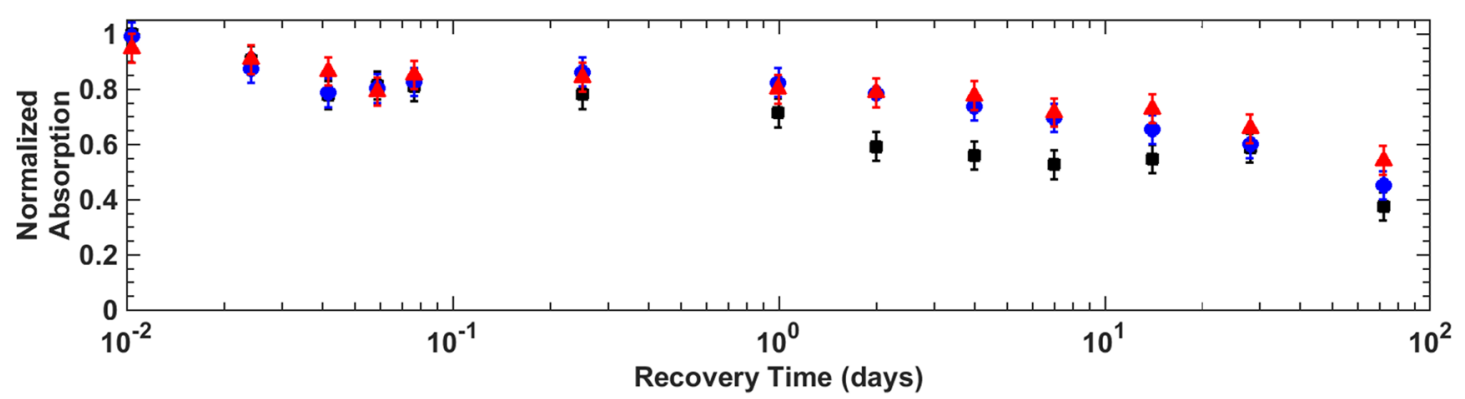

Fig. 2 a Full spectra measured for a set of samples kept at RT and highlighted region of interest for analysis. Inset graph depicts exclusively the frequency band of $\mathrm{OH}$ associated vibrations of samples during recovery period versus pristine PDMS, b normalized $\mathrm{OH}$ vibration band (3000-3700 $\mathrm{cm}^{-1}$ ) shift during 4 weeks for three sets of storage conditions, $\mathbf{c}$ normalized $\mathrm{OH}$ band max plotted as function of time for three storage temperatures. Error bars indicate the standard deviation of three measurements per data point
Fig. 3 Hydrophobic recovery of capillary filling microchannels. a Cosine of calculated dynamic advancing $\mathrm{CA}$ and corresponding Washburn constant pertaining to microchannel during recovery period, for three sets of samples stored at three different temperatures. b Schematics of the used meandering microchannel, with inlet and outlet at either ends of the long channel (a)

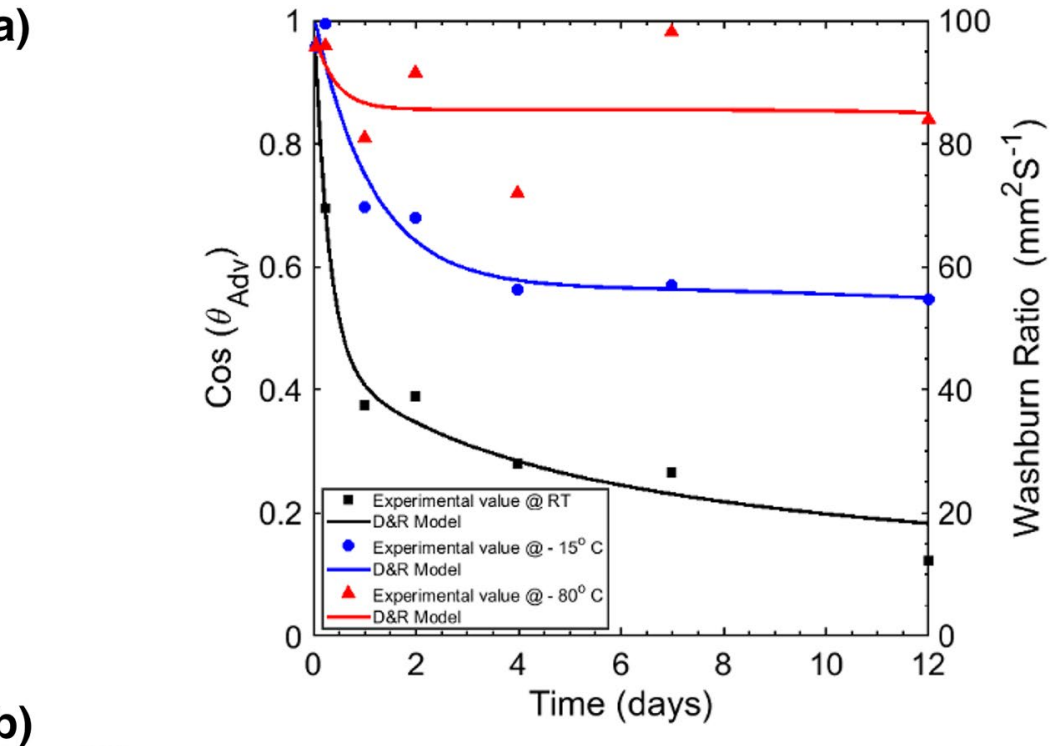

(b)

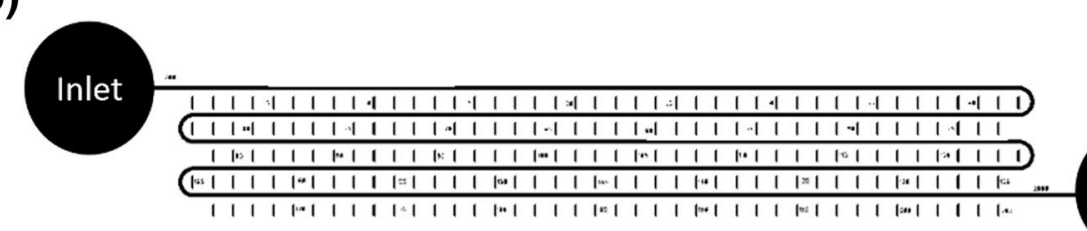


three distinct trajectories, with samples kept at $-80^{\circ}$ and RT showing least and most reduction of filling rate over time, respectively.

The results show that the colder the storage temperature, the better the initial capillary filling rate is preserved. Over the 12 days, the RT-stored samples had slowed down to $10 \mathrm{~mm}^{2} \mathrm{~s}^{-1}$, while the filling rate of the $-15{ }^{\circ} \mathrm{C}$ sample slowed down to $48 \mathrm{~mm}^{2} \mathrm{~s}^{-1}$ and the filling rate of the $-80^{\circ} \mathrm{C}$ sample slowed only slightly to $73 \mathrm{~mm}^{2} \mathrm{~s}^{-1}$ from the initial $84 \mathrm{~mm}^{2} \mathrm{~s}^{-1}$.

For a quantitative comparison to the results obtained from planar sheets (Fig. 1), we calculated the dynamic advancing contact angles from the capillary filling rates via Eq. 2 which, exhibit a similar trend of branching out to three trajectories (Fig. 3). Since the same liquid and the same cross-sectional geometry were utilized in all capillary experiments, the only parameter that changed over time and between experiments was the contact angle. Hence, from Eq. 2, we see that the capillary filling rate is directly proportional to the cosine of the advancing contact angle (Eq. 3):

$R_{\mathrm{t}} / R_{0}=\cos \left(\theta_{\mathrm{t}}\right) / \cos \left(\theta_{0}\right)$,

where $R_{0}$ is the filling rate immediately after plasma treatment, $R_{\mathrm{t}}$ is the filling rate at a specific timepoint, $\theta_{0}$ is the advancing contact angle immediately after plasma treatment and $\theta_{\mathrm{t}}$ is the calculated dynamic advancing contact angle at a specific timepoint. From the goniometer measurements of the planar sheets, the initial advancing contact angle was measured to be $7^{\circ}$, which is used as the value $\theta_{0}$ also in the case of the microchannels. A summary of how $\cos (\theta)$ ratios at various storage temperatures have evolved over time is represented in Fig. 4. Comparing the calculated microchannel advancing contact angles to the directly measured planar surface advancing contact angles reveals overall a close quantitative agreement between both types of measurements. For example, contact angle data point in the day 2 after the plasma treatment is around $41^{\circ}$ and $47^{\circ}$ for flat and channel samples, respectively, for $-15^{\circ} \mathrm{C}$ storage conditions. Similarly, for samples kept at $-80^{\circ} \mathrm{C}$ at day 12 , contact angles of $33^{\circ}$ and $35^{\circ}$ have been recorded from direct measurements and calculations, respectively. It can be seen that the CAs calculated based on the filling rate are systematically slightly higher than those of the goniometer measurements, which when taken into cosine values exhibit a lower value with respect to planar surface values (Fig. 4). A possible explanation is dynamic CA being different at higher liquid velocities (Siebold et al. 2000).

\subsection{Capillary flow pinning on geometrical features}

Re-entrant geometrical changes alongside the channel can act as metastable pinning sites for meniscus due to the increased area and the resulting added surface free energy of the expanding liquid-vapor interface. Such an energy barrier can manifest as a threshold pressure and is described by Eq. 4 . The energy barrier for a $2 \mathrm{D}$ meniscus model has been studied by Man et al. (1998). The increased liquid-gas surface energy of a widening channel causes a pressure barrier given by Eq. 4 .
Fig. 4 Hydrophobic recovery measured on planar sheets compared to hydrophobic recovery calculated from microchannel filling rates. The $\cos \left(\theta_{t}\right)$ tends to decrease as a function of recovery time, more so the higher the temperatures
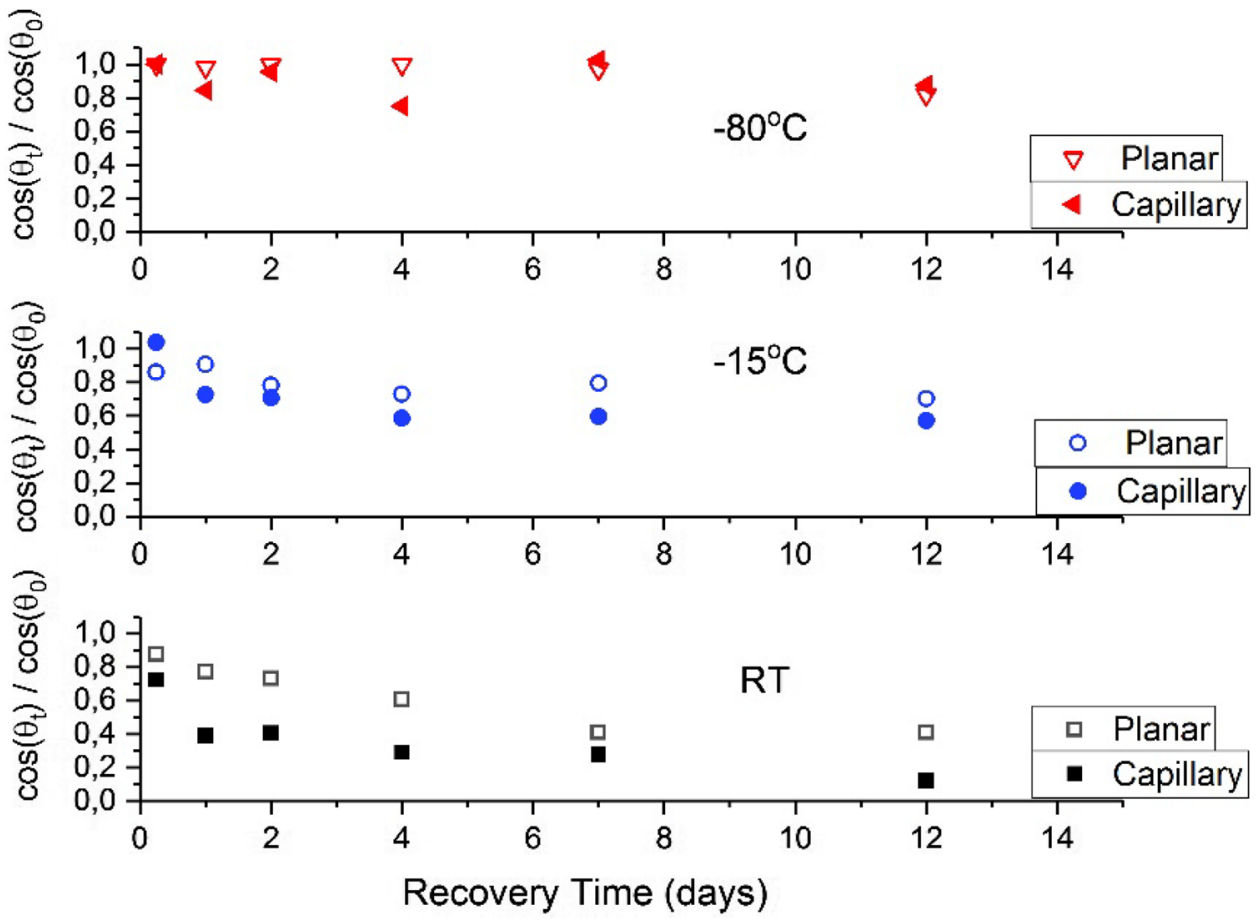
$P=-\frac{d U_{T}}{d V_{l}}=\gamma_{l a}\left(\cos _{C} \frac{d A_{s l}}{d V_{l}}-\frac{d A_{l a}}{d V_{l}}\right)$.

Re-entrant structures are common in microfluidic channels even when not specifically designed. They occur with channel intersections and changes in the cross-sectional shape. In addition to these, surface roughness of the microchannel can act as potential pinning sites. For reliable capillary filling, it is, therefore, important that the liquid is able to bypass geometrical pinning sites. In fact it is known that capillary stop valves are unreliable at lower contact angles (Duffy et al. 1999).

The advancement of the meniscus through a geometrical valve requires firstly a pivoting of curvature at the wedge point, followed by establishing an equilibrium contact angle with the slanted wall. Expansion of Eq. 4 at this point for the system predicates the meniscus curvature to fall within the interval of $\pi / 2-\theta_{\mathrm{c}} \geq \alpha \geq \pi / 2-\theta_{\mathrm{c}}-\beta$ (Fig. 5a-c). Therefore, depending on the $\beta$, the curvature $\alpha$ could render a negative value, preventing further movement. A zero or positive value of $\alpha$ would be minimum requirement of bypassing which in this cases leads to $\beta=\pi / 2-\theta_{\mathrm{c}}$. This means $P=0$ (flat meniscus) in Eq. 4, from which follows:

$\frac{d A_{l a}}{d A_{s l}}=\cos \theta_{c}=\sin \left(\pi / 2-\theta_{c}\right)=\sin \beta$.

It was our hypothesis that the hydrophobic recovery of a PDMS microchannel would not only cause a slowdown of the filling rate, but also diminish the ability of the flow to bypass pinning sites. Our empirical data on the ability of capillary flow to bypass five different geometrical valves (increasing $\beta$ ) measured at selected days during recovery, as a function of storage temperature, are shown in Table 1. The RT-stored samples eventually lost their ability to bypass the $80^{\circ}$ valve at day 7 , which corresponds to advancing contact angle $68^{\circ}$ (from Fig. 1). Samples stored at $-15{ }^{\circ} \mathrm{C}$ and $-80{ }^{\circ} \mathrm{C}$ maintained their ability to bypass all the test valves for the entire 12 days span of experiment. A snapshot of a flow that is able to bypass all obstacles is shown in Fig. $5 \mathrm{~d}$.

Comparing experimental data to the condition given by Eq. 4, it is noticeable that the flow is able to bypass geometrical obstacles better than predicted by Eq. 4. This deviation can be explained by an unaccounted for precursor flow in the model, which is observed in our experiments (Fig. 5d) and also by the 2D nature of the model presented by Leu and Chang (2004) developed a 3D model and showed that the pressure drop for 3D meniscus (in valves up to $\beta=50^{\circ}$ ) is much less than what the $2 \mathrm{D}$ model would predict.

Table 1 Ability of capillary flow to bypass a geometrical valve

\begin{tabular}{llllll}
\hline & $\beta=90^{\circ}$ & $\beta=80^{\circ}$ & $\beta=60^{\circ}$ & $\beta=40^{\circ}$ & $\beta=10^{\circ}$ \\
\hline RT $\left(22^{\circ} \mathrm{C}\right)$ & $\begin{array}{c}\text { Pinned at } \\
\text { day 7 }\end{array}$ & $\begin{array}{c}\text { Pinned at } \\
\text { day 7 }\end{array}$ & NP & NP & NP \\
$-15^{\circ} \mathrm{C}$ & NP & NP & NP & NP & NP \\
$-80^{\circ} \mathrm{C}$ & NP & NP & NP & NP & NP \\
\hline
\end{tabular}

Table cells with NP indicate valve never pinned the flow for entire span of experiments (12 days)
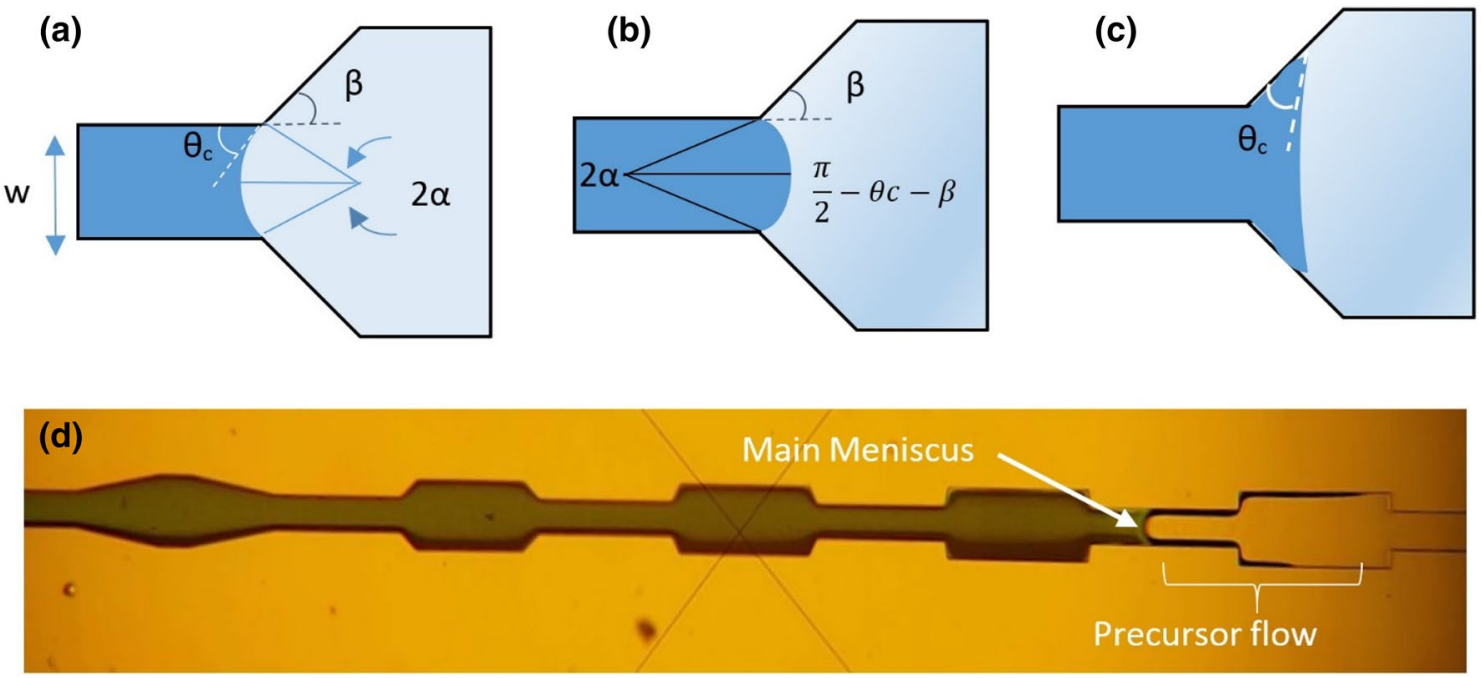

Fig. 5 Capillary flow pinning on geometrical features. a Positive curvature of the meniscus during advancement, $\mathbf{b}$ pivoting and change of the curvature at the wedge, $\mathbf{c}$ attaining equilibrium contact angle by meniscus after wedge and further expansion. d A snapshot of capil- lary flow that is able to bypass all five consecutive geometrical valves with increasing wedge angle. The experiment shown is with advancing contact angle $7^{\circ}$ right after hydrophilization 


\subsection{Discussion}

Different experiments (contact angle, capillary fill rate, capillary valve bypass and IR spectroscopy) in this work together clearly show that the hydrophilicity of plasmaoxidized PDMS can be retained over long periods of time if the recovery process is retarded by storage in a cold temperature. Direct measurements on flat sheets of PDMS over 100 days for samples stored at various temperatures single out the effect of thermally activated processes responsible for the dynamic CAs recovery, while other variables remain constant. PDMS samples stored at $-80^{\circ} \mathrm{C}$ were nearly completely wetting after 100 days and based on the trend they would likely have remained hydrophilic for much longer than that. From diffusion and reorientation model, it is predicted that the same degree of recovery happens in 1.55 , 97 and 3400 days for samples stored at RT, $-15^{\circ} \mathrm{C}$ and $-80^{\circ} \mathrm{C}$, respectively. We can confirm the 1.55 and 97 days predictions from the $\mathrm{RT}$ and $-15{ }^{\circ} \mathrm{C}$ data empirically as it is within the measurement span. Also from fitted trend lines in Fig. 1, an initial decrease in cos values is observed which occurs during a distinct relaxation time (depending on the storage temperature). Afterwards, there is a plateau stabilization for each temperature which suggests that the recovery has reached an equilibrium in one of its fastest main constituent processes, and further recovery may have shifted to other slower mechanisms. In filling experiments where the measurements capture the capillary flow kinetics, capillary filling rate was only slightly lowered from $83 \mathrm{~mm}^{2} \mathrm{~s}^{-1}$ to $73 \mathrm{~mm}^{2} \mathrm{~s}^{-1}$ when stored at $-80{ }^{\circ} \mathrm{C}$. The underlying slowdown of meniscus filling over many days in different chips is in par with the thermal dependence of recovery and also in line with final remnant of hydrophilicity of flat sheet PDMS experiments (90\% remaining for $-80{ }^{\circ} \mathrm{C}$ storage, $60 \%$ for $-15{ }^{\circ} \mathrm{C}$ and around $20 \%$ of original hydrophilicity for RT-stored samples). Meanwhle, it is noticed that the recovery rate of the microchannels seems to be relatively faster than the flat sheets. This observation may be explained by the fact that bulk source of diffusing oligomers is much more available per surface area in the case of 4-walled PDMS channels compared to the single PDMS sheet. Vibrational spectroscopy analysis used in the study traces the presence of the $\mathrm{OH}$ - bonds, a direct contributor to the surface hydrophilicity. Radicals containing $\mathrm{OH}-$ within the polymer chains created during plasma treatment remain relatively stable and unreacted when reaction energy barrier is unfulfilled (López-García 2019). We ascribe the reduced recovery rate in the cold temperatures predominantly to the reduced rate of thermally active diffusion and reorientation of the LMW species to and from surface, which are known to play a key role in surface recovery and restructuring (Hillborg and Gedde 1998; Kim et al. 2006). This is supported by the ATR-FTIR results showing more hydroxyl and carboxyl groups remaining at or near the surface over time when the samples were stored at colder temperatures. Duration of the data collection was realized based on enough time to capture the nature of the temperature dependence and plateau saturation which has been observed in other experiments.

\section{Conclusion}

The introduced cold storage method of PDMS material and microchips is shown to perform a near complete preservation of hydrophilicity if cold enough storage temperatures, e.g. $-80^{\circ} \mathrm{C}$ and lower are used. In terms of simplicity and effectiveness, cold storage compares favorably to other known strategies for postponing the hydrophobic recovery of PDMS. The method also leaves no known physical and chemical footprint regarding the properties of PDMS, and does not require any additional step of post- or pre-plasma modifications. The method is very easily adaptable since storage at $-15^{\circ}$ should be available at all laboratories. Cold storage could be an especially attractive method to retain capillary filling properties of microfluidic chips for applications such as passive lab on chip and point of care diagnostics that have preloaded biological ingredients (Hitzbleck et al. 2011), which would be preserved simultaneously in the freezer.

Acknowledgements Open access funding provided by Aalto University. The authors acknowledge funding from the Academy of Finland (\#297 360 + 272 619) and The Finnish Funding Agency for Innovation (\#211679). The work utilized the facilities of Micronova, Centre for Micro and Nanotechnology.

Open Access This article is distributed under the terms of the Creative Commons Attribution 4.0 International License (http://creativeco mmons.org/licenses/by/4.0/), which permits unrestricted use, distribution, and reproduction in any medium, provided you give appropriate credit to the original author(s) and the source, provide a link to the Creative Commons license, and indicate if changes were made.

\section{References}

Bacharouche J, Haidara H, Kunemann P, Vallat M-F, Roucoules V (2013) Singularities in hydrophobic recovery of plasma treated polydimethylsiloxane surfaces under non-contaminant atmosphere. Sens Actuators Phys 197:25-29

Bauer W-AC, Fischlechner M, Abell C, Huck WTS (2010) Hydrophilic PDMS microchannels for high-throughput formation of oil-inwater microdroplets and water-in-oil-in-water double emulsions. Lab Chip 10(14):1814-1819

Bodas D, Khan-Malek C (2006) Formation of more stable hydrophilic surfaces of PDMS by plasma and chemical treatments. Microelectron Eng 83(4):1277-1279

de Gennes P-G (2004) Capillarity and wetting phenomena-drops, bubbles, Springer. http://www.springer.com/gp/book/9780387005 928. Accessed 13 Sep 2017. (Online) 
Delamarche E, Donzel C, Kamounah FS, Wolf H, Geissler M, Stutz R, Schmidt-Winkel P, Michel B, Mathieu HJ, Schaumburg K (2003) Microcontact printing using poly(dimethylsiloxane) stamps hydrophilized by poly(ethylene oxide) silanes. Langmuir 19(21):87498758. https://doi.org/10.1021/la034370n

Duffy DC, Gillis HL, Lin J, Sheppard NF, Kellogg GJ (1999) Microfabricated centrifugal microfluidic systems: characterization and multiple enzymatic assays. Anal Chem 71(20):4669-4678

Duncombe TA, Tentori AM, Herr AE (2015) Microfluidics: reframing biological enquiry. Nat Rev Mol Cell Biol 16(9):554-567

Eddington DT, Puccinelli JP, Beebe DJ (2006) Thermal aging and reduced hydrophobic recovery of polydimethylsiloxane. Sens Actuators B Chem 114(1):170-172

Elvira KS, i Solvas XC, Wootton RCR, deMello AJ (2013) The past, present and potential for microfluidic reactor technology in chemical synthesis. Nat Chem 5(11):905-915

Ferreira P, Alves P, Coimbra P, Gil MH (2015) Improving polymeric surfaces for biomedical applications: a review. J Coat Technol Res 12(3):463-475

Hemmilä S, Cauich-Rodríguez JV, Kreutzer J, Kallio P (2012) Rapid, simple, and cost-effective treatments to achieve long-term hydrophilic PDMS surfaces. Appl Surf Sci 258(24):9864-9875

Hillborg H, Gedde UW (1998) Hydrophobicity recovery of polydimethylsiloxane after exposure to corona discharges. Polymer 39(10):1991-1998

Hillborg H, Ankner JF, Gedde UW, Smith GD, Yasuda HK, Wikström K (2000) Crosslinked polydimethylsiloxane exposed to oxygen plasma studied by neutron reflectometry and other surface specific techniques. Polymer 41(18):6851-6863

Hillborg H, Tomczak N, Olàh A, Schönherr H, Vancso GJ (2004) Nanoscale hydrophobic recovery: a chemical force microscopy study of UV/ozone-treated cross-linked poly(dimethylsiloxane). Langmuir 20(3):785-794

Hitzbleck M, Gervais L, Delamarche E (2011) Controlled release of reagents in capillary-driven microfluidics using reagent integrators. Lab Chip 11(16):2680-2685

Jokinen V, Franssila S (2008) Capillarity in microfluidic channels with hydrophilic and hydrophobic walls. Microfluid Nanofluidics 5(4):443-448

Jokinen V, Suvanto P, Franssila S (2012) Oxygen and nitrogen plasma hydrophilization and hydrophobic recovery of polymers. Biomicrofluidics 6(1):016501-1650110. https://doi. org/10.1063/1.3673251

Juncker D et al (2002) Autonomous microfluidic capillary system. Anal Chem 74(24):6139-6144

Khorasani MT, Mirzadeh H, Irani S (2008) Plasma surface modification of poly (1-lactic acid) and poly (lactic-co-glycolic acid) films for improvement of nerve cells adhesion. Radiat Phys Chem 77(3):280-287

Kim J, Chaudhury MK, Owen MJ (1999) Hydrophobicity loss and recovery of silicone HV insulation. IEEE Trans Dielectr Electr Insul 6(5):695-702

Kim J, Chaudhury MK, Owen MJ, Orbeck T (2001a) The mechanisms of hydrophobic recovery of polydimethylsiloxane elastomers exposed to partial electrical discharges. J Colloid Interface Sci 244(1):200-207

Kim J, Chaudhury MK, Owen MJ, Orbeck T (2001b) The mechanisms of hydrophobic recovery of polydimethylsiloxane elastomers exposed to partial electrical discharges. J Colloid Interface Sci 244(1):200-207

Kim J, Chaudhury MK, Owen MJ (2006) Modeling hydrophobic recovery of electrically discharged polydimethylsiloxane elastomers. J Colloid Interface Sci 293(2):364-375

Lee JN, Park C, Whitesides GM (2003) Solvent compatibility of poly(dimethylsiloxane)-based microfluidic devices. Anal Chem 75(23):6544-6554
Leu T-S, Chang P-Y (2004) Pressure barrier of capillary stop valves in micro sample separators. Sens Actuators Phys 115(2):508-515

López-García J (2019) Chapter 10—wettability analysis and water absorption studies of plasma activated polymeric materials. In: Thomas S, Mozetič M, Cvelbar U, Špatenka P, Praveen KM (eds) Non-thermal plasma technology for polymeric materials. Elsevier, Amsterdam, pp 261-285

Man PF, Mastrangelo CH, Burns MA, Burke DT (1998) Microfabricated capillarity-driven stop valve and sample injector. In: Proceedings MEMS 98. IEEE. Eleventh Annual International Workshop on micro electro mechanical systems. An investigation of micro structures, sensors, actuators, machines and systems. Cat. no. $98 \mathrm{CH} 36176$, pp 45-50

Morra M, Occhiello E, Marola R, Garbassi F, Humphrey P, Johnson D (1990) On the aging of oxygen plasma-treated polydimethylsiloxane surfaces. J Colloid Interface Sci 137(1):11-24

Mortazavi M, Nosonovsky M (2012) A model for diffusion-driven hydrophobic recovery in plasma treated polymers. Appl Surf Sci 258(18):6876-6883

Mosadegh B et al (2010) Integrated elastomeric components for autonomous regulation of sequential and oscillatory flow switching in microfluidic devices. Nat Phys 6(6):433-437

Nguyen L (2014) Minimizing hydrophobic recovery of polydimethylsiloxane after oxygen plasma treatment. Thesis

Nguyen L et al (2014) Simple and improved approaches to long-lasting, hydrophilic silicones derived from commercially available precursors. ACS Appl Mater Interfaces 6(24):22876-22883

Owen MJ, Smith PJ (1994) Plasma treatment of polydimethylsiloxane. J Adhes Sci Technol 8(10):1063-1075

Ren X, Bachman M, Sims C, Li GP, Allbritton N (2001) Electroosmotic properties of microfluidic channels composed of poly(dimethylsiloxane). J Chromatogr B Biomed Sci App 762(2):117-125

Sackmann EK, Fulton AL, Beebe DJ (2014) The present and future role of microfluidics in biomedical research. Nat Lond 507(7491):181-189

Safavieh R, Juncker D (2013) Capillarics: pre-programmed, self-powered microfluidic circuits built from capillary elements. Lab Chip 13(21):4180-4189

Siebold A, Nardin M, Schultz J, Walliser A, Oppliger M (2000) Effect of dynamic contact angle on capillary rise phenomena. Colloids Surf Physicochem Eng Asp 161(1):81-87

Tan SH, Nguyen N-T, Chua YC, Kang TG (2010) Oxygen plasma treatment for reducing hydrophobicity of a sealed polydimethylsiloxane microchannel. Biomicrofluidics 4(3):32204

Toth A, Bertoti I, Blazso M, Banhegyi G, Bognar A, Szaplonczay P (1994) Oxidative damage and recovery of silicone rubber surfaces. I. X-ray photoelectron spectroscopic study. J Appl Polym Sci 52(9):1293-1307

Tsougeni K, Petrou PS, Tserepi A, Kakabakos SE, Gogolides E (2009) Nano-texturing of poly(methyl methacrylate) polymer using plasma processes and applications in wetting control and protein adsorption. Microelectron Eng 86(4):1424-1427

Vickers JA, Caulum MM, Henry CS (2006) Generation of hydrophilic poly(dimethylsiloxane) for high-performance microchip electrophoresis. Anal Chem 78(21):7446-7452

Vlachopoulou M-E, Petrou PS, Kakabakos SE, Tserepi A, Beltsios K, Gogolides E (2009) Effect of surface nanostructuring of PDMS on wetting properties, hydrophobic recovery and protein adsorption. Microelectron Eng 86(4):1321-1324

Washburn EW (1921) The dynamics of capillary flow. Phys Rev 17(3):273-283

Xia Y, Whitesides GM (1998) Soft lithography. Annu Rev Mater Sci 28(1):153-184

Yao M, Fang J (2012) Hydrophilic PEO-PDMS for microfluidic applications. J Micromech Microeng 22(2):025012 
Yoshida S, Hagiwara K, Hasebe T, Hotta A (2013) Surface modification of polymers by plasma treatments for the enhancement of biocompatibility and controlled drug release. Surf Coat Technol 233:99-107

Zhao LH, Lee J, Sen PN (2012) Long-term retention of hydrophilic behavior of plasma treated polydimethylsiloxane (PDMS) surfaces stored under water and Luria-Bertani broth. Sens Actuators Phys $181: 33-42$
Publisher's Note Springer Nature remains neutral with regard to jurisdictional claims in published maps and institutional affiliations. 